\title{
Numeracy, Health Literacy, Cognition, and 30-Day Readmissions among Patients with Heart Failure
}

\author{
Madeline R. Sterling, MD, MPH ${ }^{1,2 *}$, Monika M. Safford, MD ${ }^{1,2}$, Kathryn Goggins, MPH ${ }^{3,4,5}$, \\ Sam K. Nwosu, MS ${ }^{6}$, Jonathan S. Schildcrout, $\mathrm{PhD}^{6}$, Kenneth A. Wallston, $\mathrm{PhD}^{7}$, \\ Amanda S. Mixon, MD, MS, MSPH, FHM $33,8,9$, Russell L. Rothman, MD, MPP 3,8 , Sunil Kripalani, MD, MSc, SFHM $3,4,5,8$ \\ for the Vanderbilt Inpatient Cohort Study (VICS)
}

\begin{abstract}
'Department of Medicine, Weill Cornell Medical College, New York, New York; 2Division of General Internal Medicine, Weill Cornell Medical College, New York, New York; ${ }^{3}$ Center for Health Services Research, Vanderbilt University Medical Center, Nashville, Tennessee; ${ }^{4}$ Center for Effective Health Communication, Vanderbilt University Medical Center, Nashville, Tennessee; ${ }^{5}$ Center for Clinical Quality and Implementation Research, Vanderbilt University Medical Center, Nashville, Tennessee; ' ${ }^{6}$ epartment of Biostatistics, Vanderbilt University Medical Center, Nashville, Tennessee; ${ }^{7}$ School of Nursing, Vanderbilt University, Nashville, Tennessee; ${ }^{8}$ Division of General Internal Medicine and Public Health, Department of Medicine, Vanderbilt University, Nashville, Tennessee; ${ }^{9}$ Department of Veterans Affairs, Tennessee Valley Healthcare System Geriatric Research Education and Clinical Center (GRECC), Nashville, Tennessee.
\end{abstract}

BACKGROUND: Numeracy, health literacy, and cognition are important for chronic disease management. Prior studies have found them to be associated with poorer selfcare and worse clinical outcomes, but limited data exists in the context of heart failure (HF), a condition that requires patients to monitor their weight, fluid intake, and dietary salt, especially in the posthospitalization period.

OBJECTIVE: To examine the relationship between numeracy, health literacy, and cognition with 30-day readmissions among patients hospitalized for acute decompensated HF (ADHF).

DESIGN/SETTING/PATIENTS: The Vanderbilt Inpatient Cohort Study is a prospective longitudinal study of adults hospitalized with acute coronary syndromes and/or ADHF. We studied 883 adults hospitalized with ADHF.

MEASUREMENTS: During their hospitalization, a baseline interview was performed in which demographic characteristics, numeracy, health literacy, and cognition were assessed.
Through chart review, clinical characteristics were determined. The outcome of interest was 30-day readmission to any acute care hospital. To examine the association between numeracy, health literacy, cognition, and 30-day readmissions, multivariable Poisson (log-linear) regression was used.

RESULTS: Of the 883 patients admitted for ADHF, 23.8\% $(n=210)$ were readmitted within 30 days; $33.9 \%$ of the study population had inadequate numeracy skills, $24.6 \%$ had inadequate/marginal literacy skills, and $53 \%$ had any cognitive impairment. Numeracy and cognition were not associated with 30-day readmissions. Though (objective) health literacy was associated with 30-day readmissions in unadjusted analyses, it was not in adjusted analyses.

CONCLUSIONS: Numeracy, health literacy, and cognition were not associated with 30 -day readmission among this sample of patients hospitalized with ADHF. Journal of Hospital Medicine 2018;13:145-151. Published online first February 12, 2018. (@) 2018 Society of Hospital Medicine

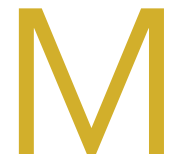

ost studies to identify risk factors for readmission among patients with heart failure (HF) have focused on demographic and clinical characteristics. ${ }^{1,2}$ Although easy to extract from administrative databases, this approach fails to capture the complex psychosocial and cognitive factors that influence the ability

\footnotetext{
*Address for correspondence: Madeline R. Sterling, MD, MPH, AHRQ Health Services Research Fellow, Division of General Internal Medicine, Department of Medicine, Weill Cornell Medical College, 1300 York Avenue, P.O. Box 46, New York, NY 10065; Telephone: 646-962-5029; Fax: 646-962-0621; E-mail: mrs9012@ med.cornell.edu

Additional Supporting Information may be found in the online version of this article.
}

Received: June 5, 2017; Revised: October 24, 2017;

Accepted: November 11, 2017

2018 Society of Hospital Medicine DOI 10.12788/jhm.2932 of HF patients to manage their disease in the postdischarge period, as depicted in the framework by Meyers et al. ${ }^{3}$ (2014). To date, studies have found low health literacy, decreased social support, and cognitive impairment to be associated with health behaviors and outcomes among HF patients, including decreased self-care, ${ }^{4}$ low HF-specific knowledge, ${ }^{5}$ medication nonadherence, ${ }^{6}$ hospitalizations, ${ }^{7}$ and mortality. ${ }^{8-10}$ Less, however, is known about the effect of numeracy on HF outcomes, such as 30-day readmission.

Numeracy, or quantitative literacy, refers to the ability to access, understand, and apply numerical data to health-related decisions..$^{11}$ It is estimated that 110 million people in the United States have limited numeracy skills. ${ }^{12}$ Low numeracy is a risk factor for poor glycemic control among patients with diabetes,$^{13}$ medication adherence in HIV/AIDS, ${ }^{14}$ and worse blood pressure control in hypertensives. ${ }^{15}$ Much like these conditions, HF requires that patients understand, use, and act on numerical information. Maintaining a low-salt diet, monitoring 
weight, adjusting diuretic doses, and measuring blood pressure are tasks that HF patients are asked to perform on a daily or near-daily basis. These tasks are particularly important in the posthospitalization period and could be complicated by medication changes, which might create additional challenges for patients with inadequate numeracy. Additionally, cognitive impairment, which is a highly prevalent comorbid condition among adults with $\mathrm{HF}^{16,17}$ might impose additional barriers for those with inadequate numeracy who do not have adequate social support. However, to date, numeracy in the context of HF has not been well described.

Herein, we examined the effects of numeracy, alongside health literacy and cognition, on 30-day readmission risk among patients hospitalized for acute decompensated HF (ADHF).

\section{METHODS}

\section{Study Design}

The Vanderbilt Inpatient Cohort Study (VICS) is a prospective observational study of patients admitted with cardiovascular disease to Vanderbilt University Medical Center (VUMC), an academic tertiary care hospital. VICS was designed to investigate the impact of social determinants of health on postdischarge health outcomes. A detailed description of the study rationale, design, and methods is described elsewhere. ${ }^{3}$

Briefly, participants completed a baseline interview while hospitalized, and follow-up phone calls were conducted within 1 week of discharge, at 30 days, and at 90 days. At 30 and 90 days postdischarge, healthcare utilization was ascertained by review of medical records and patient report. Clinical data about the index hospitalization were also abstracted. The Vanderbilt University Institutional Review Board approved the study.

\section{Study Population}

Patients hospitalized from 2011 to 2015 with a likely diagnosis of acute coronary syndrome and/or ADHF, as determined by a physician's review of the medical record, were identified as potentially eligible. Research assistants assessed these patients for the presence of the following exclusion criteria: less than 18 years of age, non-English speaking, unstable psychiatric illness, a low likelihood of follow-up (eg, no reliable telephone number), on hospice, or otherwise too ill to complete an interview. Additionally, those with severe cognitive impairment, as assessed from the medical record (such as seeing a note describing dementia), and those with delirium, as assessed by the brief confusion assessment method, were excluded from enrollment in the study. ${ }^{18,19}$ Those who died before discharge or during the 30-day follow-up period were excluded. For this analysis, we restricted our sample to only include participants who were hospitalized for ADHF.

\section{Outcome Measure: 30-Day Readmission}

The main outcome was all-cause readmission to any hospital within 30 days of discharge, as determined by patient interview, review of electronic medical records from VUMC, and review of outside hospital records.
Main Exposures: Numeracy, Health Literacy, and Cognitive Impairment

Numeracy was assessed with a 3-item version of the Subjective Numeracy Scale (SNS-3), which quantifies the patients perceived quantitative abilities. ${ }^{20}$ Other authors have shown that the SNS-3 has a correlation coefficient of 0.88 with the fulllength SNS-8 and a Cronbach's alpha of $0.78 .{ }^{20-22}$ The SNS-3 is reported as the mean on a scale from 1 to 6 , with higher scores reflecting higher numeracy.

Subjective health literacy was assessed by using the 3-item Brief Health Literacy Screen (BHLS). ${ }^{23}$ Scores range from 3 to 15, with higher scores reflecting higher literacy. Objective health literacy was assessed with the short form of the Test of Functional Health Literacy in Adults (sTOFHLA). ${ }^{24,25}$ Scores may be categorized as inadequate (0-16), marginal (17-22), or adequate (23-36).

We assessed cognition by using the 10-item Short Portable Mental Status Questionnaire (SPMSQ). ${ }^{26}$ The SPMSQ, which describes a person's capacity for memory, structured thought, and orientation, has been validated and has demonstrated good reliability and validity. ${ }^{27}$ Scores of 0 were considered to reflect intact cognition, and scores of 1 or more were considered to reflect any cognitive impairment, a scoring approach employed by other authors. ${ }^{28}$ We used this approach, rather than the traditional scoring system developed by Pfeiffer et al. ${ }^{26}$ (1975), because it would be the most sensitive to detect any cognitive impairment in the VICS cohort, which excluded those with severe cognition impairment, dementia, and delirium.

\section{Covariates}

During the hospitalization, participants completed an in-person interviewer-administered baseline assessment composed of demographic information, including age, self-reported race (white and nonwhite), educational attainment, home status (married, not married and living with someone, not married and living alone), and household income.

Clinical and diagnostic characteristics abstracted from the medical record included a medical history of HF, HF subtype (classified by left ventricular ejection fraction [LVEF]), coronary artery disease, chronic obstructive pulmonary disease (COPD), diabetes mellitus (DM), and comorbidity burden as summarized by the van Walraven-Elixhauser score. ${ }^{29,30}$ Depressive symptoms were assessed during the 2 weeks prior to the hospitalization by using the first 8 items of the Patient Health Questionnaire. ${ }^{31}$ Scores ranged from 0 to 24, with higher scores reflecting more severe depressive symptoms. Laboratory values included estimated glomerular filtration rate (eGFR), hemoglobin ( $/ \mathrm{dll}$ ), sodium (mg/L), and brain natriuretic peptide (BNP) $(\mathrm{pg} / \mathrm{ml})$ from the last laboratory draw before discharge. Smoking status was also assessed (current and former/nonsmokers).

Hospitalization characteristics included length of stay in days, number of prior admissions in the last year, and transfer to the intensive care unit during the index admission.

\section{Statistical Analysis}

Descriptive statistics were used to summarize patient characteristics. The Kruskal-Wallis test and the Pearson $\chi^{2}$ test were 
used to determine the association between patient characteristics and levels of numeracy, literacy, and cognition separately. The unadjusted relationship between patient characteristics and 30-day readmission was assessed by using Wilcoxon rank sums tests for continuous variables and Pearson $\chi^{2}$ tests for categorical variables. In addition, a correlation matrix was performed to assess the correlations between numeracy, health literacy, and cognition (supplementary Figure 1).

To examine the association between numeracy, health literacy, and cognition and 30-day readmissions, a series of multivariable Poisson (log-linear) regression models were fit. ${ }^{32}$ Like other studies, numeracy, health literacy, and cognition were examined as categorical and continuous measures in models. ${ }^{33}$ Each model was modified with a sandwich estimator for robust standard errors. Log-linear models were chosen over logistic regression models for ease of interpretation because (exponentiated) parameters correspond to risk ratios (RRs) as opposed to odds ratios. Furthermore, the fitting challenges as sociated with log-linear models when predicted probabilities are near 0 or 1 were not present in these analyses. Redundancy analyses were conducted to ensure that independent variables were not highly correlated with a linear combination of the other independent variables. To avoid case-wise deletion of records with missing covariates, we employed multiple imputation with 10 imputation samples by using predictive mean matching. ${ }^{34,35}$ All analyses were conducted in $\mathrm{R}$ version 3.1.2 (The R Foundation, Vienna, Austria). ${ }^{36}$

\section{RESULTS}

Overall, 883 patients were included in this analysis (supplementary Figure 2). Of the 883 participants, $46 \%$ were female and $76 \%$ were white (Table 1). Their median age was 60 years (interdecile range [IDR] 39-78) and the median educational attainment was 13.5 years (IDR 11-18).

Characteristics of the study sample by levels of subjective numeracy, objective health literacy, and cognition are shown in Table 1. A total of $33.9 \%$ had inadequate health numeracy (SNS scores 1-3 on a scale of 1-6) with an overall mean subjective numeracy score of 4.3 (standard deviation \pm 1.3 ). Patients with inadequate numeracy were more likely to be women, nonwhite, and have lower education and income. Overall, $24.6 \%$ of the study population had inadequate/marginal objective health literacy, which is similar to the $26.1 \%$ with inadequate health literacy by the subjective literacy scale (BHLS scores 3-9 on a scale of 3-15) (supplementary Table 1). Patients with inadequate objective health literacy were more likely to be older, nonwhite, have less education and income, and more comorbidities compared with those with marginal/adequate health literacy. Overall, 53\% of participants had any cognitive impairment (SPMSO score=1 or greater). They were more likely to be older, female, have less education and income, a greater number of comorbidities, and a higher severity of HF during the index admission compared with those with intact cognition.

A total of $23.8 \%(n=210)$ of patients were readmitted within 30 days of discharge (Table 2). There was no statistically sig- nificant difference in readmission by numeracy level $(P=.66)$. Readmitted patients were more likely to have lower objective health literacy compared with those who were not readmitted (27.1 vs 28.3; $P=.04$ ). A higher percentage of readmitted patients were cognitively impaired (57\%) compared with those not readmitted (51\%); however, this difference was not statistically significant $(P=.11)$. Readmitted patients did not differ from nonreadmitted patients by demographic factors (supplementary Table 2). They were, however, more likely to have a history of HF, COPD, diabetes, CKD, higher Elixhauser scores, lower eGFR and lower sodium prior to discharge, and a greater number of prior readmissions in the last 12 months compared with those who were not readmitted (all $P<.05$ ).

In unadjusted and adjusted analyses, no statistically significant associations were seen between numeracy and the risk of 30-day readmission (Table 3). Additionally, in the adjusted analyses, there was no statistically significant association between objective health literacy or cognition and 30-day readmission. (supplementary Table 3). In a fully adjusted model, a history of diabetes was associated with a $30 \%$ greater risk of 30-day readmission compared with patients without a history of diabetes ( $R R=1.30 ; P=.04)$ (supplementary Table 3). Per a 13 -point increase in the Elixhauser score, the risk of readmission within 30 days increased by approximately $21 \%(R R=1.21$; $P=.02$ ). Additionally, having 3 prior hospital admissions in the previous 12 months was associated with a $30 \%$ higher risk of readmission than having 2 or fewer prior hospital admissions $(\mathrm{RR}=1.3 ; P<.001)$.

\section{DISCUSSION}

This is the first study to examine the effect of numeracy alongside literacy and cognition on 30-day readmission risk among patients hospitalized with ADHF. Overall, we found that $33.9 \%$ of participants had inadequate numeracy skills, and $24.6 \%$ had inadequate or marginal health literacy. In unadjusted and adjusted models, numeracy was not associated with 30-day readmission. Although (objective) low health literacy was associated with 30-day readmission in unadjusted models, it was not in adjusted models. Additionally, though 53\% of participants had any cognitive impairment, readmission did not differ significantly by this factor. Taken together, these findings suggest that other factors may be greater determinants of 30-day readmissions among patients hospitalized for ADHF.

Only 1 other study has examined the effect of numeracy on readmission risk among patients hospitalized for HF. In this multicenter prospective study, McNaughton et al. ${ }^{37}$ found low numeracy to be associated with higher odds of recidivism to the emergency department (ED) or hospital within 30 days. Our findings may differ from theirs for a few reasons. First, their study had a significantly higher percentage of individuals with low numeracy (55\%) compared with ours (33.9\%). This may be because they did not exclude individuals with severe cognitive impairment, and their patient population was of lower socioeconomic status (SES) than ours. Low SES is associated with higher 30-day readmissions among HF patients ${ }^{1,10}$ throughout the literature, and low numeracy is associated with low SES in other diseas- 
TABLE 1. Characteristics of Study Participants Hospitalized for Acute Decompensated Heart Failure (ADHF) by Subjective Numeracy, Objective Health Literacy, and Cognition in the Vanderbilt Inpatient Cohort Study (VICS)

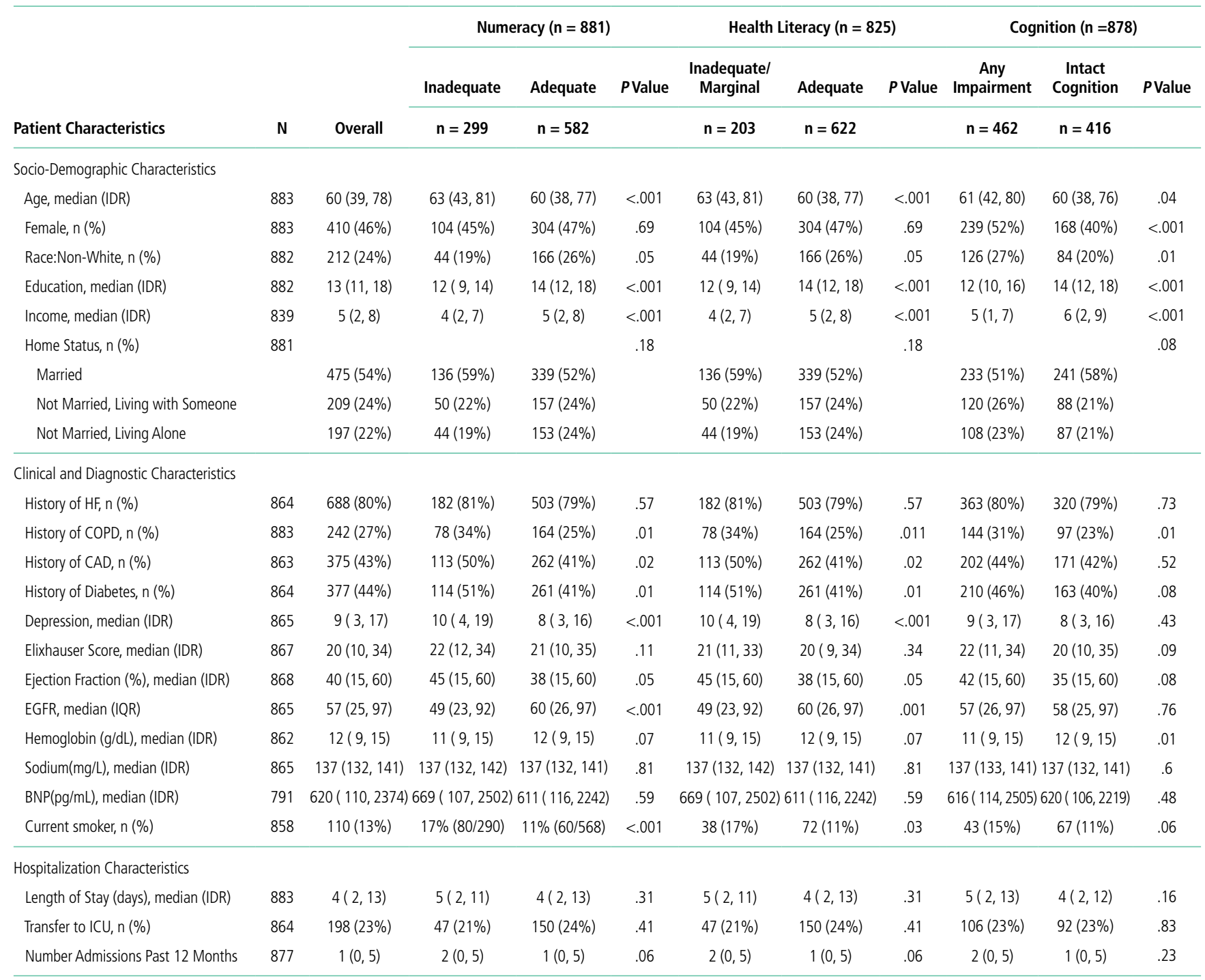

NOTE: Continuous variables are summarized with the median and interdecile range: median (IDR). Categorical variables are summarized with the $\mathrm{n}$ and percentage: $\mathrm{n}$ (\%). $\mathrm{N}$ is the number of nonmissing values. Income was considered a continuous variable, but the numbers represent ordinal categories: $1=<\$ 10,000 ; 2=\$ 10,000$ to $\$ 14,999 ; 3=\$ 15,000$ to $\$ 19,999 ; 4=\$ 20,000$ to $\$ 24,999 ; 5=\$ 25,000$ to $\$ 34,999 ; 6=\$ 35,000$ to $\$ 49,999 ; 7=\$ 50,000$ to $\$ 74,999 ; 8=\$ 75,000$ to $\$ 99,999 ; 9=\$ 100,00$. Educational attainment ranges from 1 year to 25 years. For associations with categorical variables, the Pearson $\chi^{2}$ test was used. For associations with continuous variables, the Kruskal-Wallis test was used. Abbreviations: ADHF, acute decompensated heart failure; BNP, brain natriuretic peptide; CAD, coronary artery disease; COPD, chronic obstructive pulmonary disease; eGFR, estimated glomerular filtration rate; HF, heart failure; ICU, intensive care unit; IDR, interdecile range; IQR, interquartile range; VICS, Vanderbilt Inpatient Cohort Study.

es. ${ }^{13,38,39}$ Finally, they studied recidivism, which was defined as any unplanned return to the ED or hospital within 30 days of the index ED visit for acute HF. We only focused on 30-day readmissions, which also may explain why our results differed.

We found that health literacy was not associated with 30-day readmissions, which is consistent with the literature. Although an association between health literacy and mortality exists among adults with HF, several studies have not found an association between health literacy and 30- and 90-day readmission among adults hospitalized for $\mathrm{HF}^{8,9,40}$ Although we found an association between objective health literacy and 30-day readmission in unadjusted analyses, we did not find one in the multivariable model. This, along with our numeracy finding, suggests that numeracy and literacy may not be driving the 30day readmission risk among patients hospitalized with ADHF.

We examined cognition alongside numeracy and literacy because it is a prevalent condition among HF patients and because it is associated with adverse outcomes among patients with $\mathrm{HF}$, including readmission. ${ }^{41,42}$ Studies have shown that HF preferentially affects certain cognitive domains, ${ }^{43}$ some of which are vital to HF self-care activities. We found that $53 \%$ of patients had any cognitive impairment, which is consistent with the literature of adults hospitalized for ADHF.44,45 Cognitive impairment was not, however, associated with 30-day readmissions. There 
may be a couple reasons for this. First, we measured cognitive impairment with the SPMSO, which, although widely used and well-validated, does not assess executive function, the domain most commonly affected in HF patients with cognitive impairment. ${ }^{46}$ Second, patients with severe cognitive impairment and those with delirium were excluded from this study, which may have limited our ability to detect differences in readmission by this factor.

As in prior studies, we found that a history of DM and more hospitalizations in the prior year were independently associated with 30-day readmissions in fully adjusted models. Like other studies, in adjusted models, we found that LVEF and a history of $\mathrm{HF}$ were not independently associated with 30-day readmission. ${ }^{47-49}$ This, howev$\mathrm{er}$, is not surprising because recent studies have shown that, although HF patients are at risk for multiple hospitalizations, early readmission after a hospitalization for ADHF specifically is often because of reasons unrelated to HF or a non-cardiovascular cause in general. 50,51

Although a negative study, several important themes emerged. First, while we were able to assess numeracy, health literacy, and cognition, none of these measures were HF-specific. It is possible that we did not see an effect on readmission because our instruments failed to assess domains specific to $H F$, such as monitoring weight changes, following a low-salt diet, and interpreting blood pressure. Currently, however, no HF-specific objective numeracy measure exists. With respect to health literacy, only 1 HF-specific measure exists, ${ }^{52}$ although it was only recently developed and validated. Second, while numeracy may not be a driving influence of all-cause 30-day readmissions, it may be associated with other health behaviors and quality metrics that we did not examine here, such as self-care, medication adherence, and HF-specific readmissions. Third, it is likely that the progression of HF itself, as well as the clinical management of patients following discharge, contribute significantly to 30-day readmissions. Increased attention to predischarge processes for HF patients occurred at VUMC during the study period; close follow-up and evidence-directed therapies may have mitigated some of the expected associations. Finally, we were not able to assess numeracy of participants' primary caregivers who may help patients at home, especially postdischarge. Though a number of studies have examined the role of family caregivers in the management of $\mathrm{HF}^{53,54}$ none have examined numeracy levels of caregivers in the context of HF, and this may be worth doing in future studies.

Numeracy

Health literacy

Cognition Inpatient Cohort Study.
TABLE 2. 30-Day Readmissions by Numeracy, Health Literacy, and Cognition among Participants Hospitalized for ADHF in the VICS

\begin{tabular}{ccccc}
\hline & Overall & No Readmission & Readmission & \\
\cline { 2 - 4 } $\mathrm{N}$ & $\mathrm{N}=883$ & $\mathrm{~N}=673$ & $\mathrm{~N}=210$ & PValue \\
\hline
\end{tabular}

\begin{tabular}{|c|c|c|c|c|c|}
\hline Numeracy category & 881 & & & & .75 \\
\hline Inadequate & & $299(34 \%)$ & $230(34 \%)$ & $69(33 \%)$ & \\
\hline Adequate & & $582(66 \%)$ & $442(66 \%)$ & $140(67 \%)$ & \\
\hline Numeracy score & 881 & $4(2-6)$ & $4(2-6)$ & $5(2-6)$ & .66 \\
\hline
\end{tabular}

\begin{tabular}{|c|c|c|c|c|c|}
\hline Subjective literacy category (BHLS) & 880 & & & & .67 \\
\hline Inadequate & & $230(26 \%)$ & $178(26 \%)$ & $52(25 \%)$ & \\
\hline Adequate & & $650(74 \%)$ & $494(74 \%)$ & $156(75 \%)$ & \\
\hline BHLS & 881 & $12(7-15)$ & $12(7-15)$ & $12(6-15)$ & .52 \\
\hline Objective literacy category (sTOFHLA) & 825 & & & & .11 \\
\hline Inadequate & & $127(15 \%)$ & $89(14 \%)$ & $38(19 \%)$ & \\
\hline Marginal & & $76(9 \%)$ & $63(10 \%)$ & $13(7 \%)$ & \\
\hline Adequate & & $622(75 \%)$ & $475(76 \%)$ & $147(74 \%)$ & \\
\hline STOFHLA & 825 & $32(14-36)$ & $33(15-36)$ & $31(12-35)$ & .04 \\
\hline
\end{tabular}

\begin{tabular}{|c|c|c|c|c|c|}
\hline Cognition category & 878 & & & & .11 \\
\hline Cognitive impairment & & $462(53 \%)$ & 342 (51\%) & $120(57 \%)$ & \\
\hline Intact cognition & & $416(47 \%)$ & $327(49 \%)$ & $89(43 \%)$ & \\
\hline Cognition score & 878 & $0(0-1)$ & $0(0-1)$ & $0(0-2)$ & .09 \\
\hline
\end{tabular}

NOTE: $\mathrm{N}$ is the number of nonmissing values. For associations with categorical variables, the Pearson $\chi^{2}$ test was used. For associations with continuous variables, the Wilcoxon test was used. Abbreviations: ADHF, acute decompensated heart failure; BHLS, Brief Health Literacy Screen; sTOFHLA, Short Test of Functional Health Literacy in Adults; VICS, Vanderbilt

Overall, our study has several strengths. The size of the cohort is large and there were high response rates during the follow-up period. Unlike other HF readmission studies, VICS accounts for readmissions to outside hospitals. Approximately $35 \%$ of all hospitalizations in VICS are to outside facilities. Thus, the ascertainment of readmissions to hospitals other than Vanderbilt is more comprehensive than if readmissions to VUMC were only considered. We were able to include a number of clinical comorbidities, laboratory and diagnostic tests from the index admission, and hospitalization characteristics in our analyses. Finally, we performed additional analyses to investigate the correlation between numeracy, literacy, and cognition; ultimately, we found that the majority of these correlations were weak, which supports our ability to study them simultaneously among VICS participants.

Nonetheless, we note some limitations. Although we cap- 
TABLE 3. The Effect of Numeracy on 30-Day Readmissions among Those Hospitalized for ADHF in the VICS

\begin{tabular}{|c|c|c|c|c|c|c|c|c|c|c|c|c|c|c|c|}
\hline \multirow[b]{2}{*}{ Numeracy } & \multicolumn{3}{|c|}{ Model 1} & \multicolumn{3}{|c|}{ Model 2} & \multicolumn{3}{|c|}{ Model 3} & \multicolumn{3}{|c|}{ Model 4} & \multicolumn{3}{|c|}{ Model 5} \\
\hline & RR & $95 \% \mathrm{Cl}$ & $P$ Value & RR & $95 \% \mathrm{Cl}$ & $P$ Value & RR & $95 \% \mathrm{Cl}$ & $P$ Value & RR & $95 \% \mathrm{Cl}$ & $P$ Value & RR & $95 \% \mathrm{Cl}$ & $P$ Value \\
\hline $\begin{array}{l}\text { Numeracy score } \\
\text { (per } 2 \text { point change) }\end{array}$ & 1.02 & $0.86-1.23$ & .79 & 1.09 & $0.89-1.33$ & .39 & 1.04 & $0.83-1.29$ & .75 & 1.06 & $0.85-1.33$ & .57 & 1.04 & $0.83-1.30$ & .72 \\
\hline
\end{tabular}

NOTE: Poisson Model Estimates: Model 1 adjusts for numeracy alone; Model 2 adjusts for the Model 1 variable and adjusts for health literacy and cognition; Model 3 adjusts for the Model 2 variables and demographics; Model 4 adjusts for Model 3 variables and clinical and diagnostic characteristics; and Model 5 adjusts for Model 4 variables and hospitalization characteristics. 2 variables and demographics; Model 4 adjusts for Model 3 variables and clinical and diagnostic characteristics; and Model 5 adjusts for $\mathrm{M}$
Abbreviations: ADHF, acute decompensated heart failure; Cl, confidence interval; RR, risk ratio; VICS, Vanderbilt Inpatient Cohort Study.

tured readmissions to outside hospitals, the study took place at a single referral center in Tennessee. Though patients were diverse in age and comorbidities, they were mostly white and of higher SES. Finally, we used home status as a proxy for social support, which may underestimate the support that home care workers provide.

In conclusion, in this prospective longitudinal study of adults hospitalized with ADHF, inadequate numeracy was present in more than a third of patients, and low health literacy was present in roughly a quarter of patients. Neither numeracy nor health literacy, however, were associated with 30-day readmissions in adjusted analyses. Any cognitive impairment, although present in roughly one-half of patients, was not associated with 30-day readmission either. Our findings suggest that other influences may play a more dominant role in determining 30-day readmission rates in patients hospitalized for ADHF than inadequate numeracy, low health literacy, or cognitive impairment as assessed here.

\section{References}

1. Ross JS, Mulvey GK, Stauffer B, et al. Statistical models and patient predictors of readmission for heart failure: a systematic review. Arch of Intern Med. 2008;168(13):1371-1386.

2. Zaya M, Phan A, Schwarz ER. Predictors of re-hospitalization in patients with chronic heart failure. World J Cardiol. 2012;4(2):23-30.

3. Meyers AG, Salanitro A, Wallston KA, et al. Determinants of health after hospital discharge: rationale and design of the Vanderbilt Inpatient Cohort Study (VICS). BMC Health Serv Res. 2014;14:10-19.

4. Harkness K, Heckman GA, Akhtar-Danesh N, Demers C, Gunn E, McKelvie RS. Cognitive function and self-care management in older patients with heart failure. Eur J Cardiovasc Nurs. 2014;13(3):277-284.

5. Dennison CR, McEntee ML, Samuel L, et al. Adequate health literacy is associated with higher heart failure knowledge and self-care confidence in hospitalized patients. J Cardiovasc Nurs. 2011;26(5):359-367.

6. Mixon AS, Myers AP, Leak $C L$, et al. Characteristics associated with post-discharge medication errors. Mayo Clin Proc. 2014;89(8):1042-1051.

7. Wu JR, Holmes GM, DeWalt DA, et al. Low literacy is associated with increased risk of hospitalization and death among individuals with heart failure. J Gen Intern Med. 2013;28(9):1174-1180.

8. McNaughton CD, Cawthon C, Kripalani S, Liu D, Storrow AB, Roumie CL. Health literacy and mortality: a cohort study of patients hospitalized for acute heart failure. J Am Heart Assoc. 2015;4(5):e000682. doi:10.1161/ JAHA.115.000682.

9. Moser DK, Robinson S, Biddle MJ, et al. Health Literacy Predicts Morbidity and Mortality in Rural Patients With Heart Failure. J Card Fail. 2015;21(8):612-618.

10. Calvillo-King L, Arnold D, Eubank KJ, et al. Impact of social factors on risk of readmission or mortality in pneumonia and heart failure: systematic review. $J$ Gen Intern Med. 2013;28(2):269-282.

11. Rothman RL, Montori VM, Cherrington A, Pignone MP. Perspective: the role of numeracy in health care. J Health Commun. 2008;13(6):583-595.

12. Kutner M, Greenberg E, Baer J. National Assessment of Adult Literacy: A First Look at the Literacy of America's Adults in the 21st Century. Jessup: US Department of Education National Center for Education Statistics; 2006

13. Cavanaugh $\mathrm{K}$, Huizinga MM, Wallston KA, et al. Association of numeracy and diabetes control. Ann Intern Med. 2008;148(10):737-746.

14. Ciampa PJ, Vaz LM, Blevins M, et al. The association among literacy, numer-

\section{Acknowledgments}

This research was supported by the National Heart, Lung, and Blood Institute (R01 HL109388) and in part by the National Center for Advancing Translational Sciences (UL1 TR000445-06). The content is solely the responsibility of the authors and does not necessarily represent official views of the National Institutes of Health. The authors' funding sources did not participate in the planning, collection, analysis, or interpretation of data or in the decision to submit for publication. Dr. Sterling is supported by T32HS000066 from the Agency for Healthcare Research and Quality. The content is solely the responsibility of the authors and does not necessarily represent the official views of the Agency for Healthcare Research and Quality. Dr. Mixon has a VA Health Services Research and Development Service Career Development Award at the Tennessee Valley Healthcare System, Department of Veterans Affairs (CDA 12-168). This material was presented at the Society of General Internal Medicine Annual Meeting on April 20, 2017, in Washington, DC.

Disclosure: Dr. Kripalani reports personal fees from Verustat, personal fees from SAl Interactive, and equity from Bioscape Digital, all outside of the submitted work. Dr. Rothman and Dr. Wallston report personal fees from EdLogics outside of the submitted work. All of the other authors have nothing to disclose.

acy, HIV knowledge and health-seeking behavior: a population-based survey of women in rural Mozambique. PloS One. 2012;7(6):e39391. doi:10.1371/ journal.pone.0039391.

15. Rao VN, Sheridan SL, Tuttle LA, et al. The effect of numeracy level on completeness of home blood pressure monitoring. J Clin Hypertens. 2015;17(1):39-45

16. Hanon O, Contre C, De Groote P, et al. High prevalence of cognitive disorders in heart failure patients: Results of the EFICARE survey. Arch Cardiovasc Dis Supplements. 2011;3(1):26.

17. Vogels RL, Scheltens P, Schroeder-Tanka JM, Weinstein HC. Cognitive impairment in heart failure: a systematic review of the literature. Eur $\mathrm{J}$ Heart Fail. 2007:9(5):440-449

18. Ely EW, Inouye SK, Bernard GR, et al. Delirium in mechanically ventilated patients: validity and reliability of the confusion assessment method for the intensive care unit (CAM-ICU). JAMA. 2001;286(21):2703-2710.

19. Inouye SK, van Dyck CH, Alessi CA, Balkin S, Siegal AP, Horwitz Rl. Clarifying confusion: the confusion assessment method. A new method for detection of delirium. Ann Intern Med. 1990;113(12):941-948.

20. Fagerlin A, Zikmund-Fisher BJ, Ubel PA, Jankovic A, Derry HA, Smith DM. Measuring numeracy without a math test: development of the Subjective Numeracy Scale. Med Decis Making. 2007;27(5):672-680.

21. Zikmund-Fisher BJ, Smith DM, Ubel PA, Fagerlin A. Validation of the Subjective Numeracy Scale: effects of low numeracy on comprehension of risk communications and utility elicitations. Med Decis Making. 2007;27(5): 663-671.

22. McNaughton CD, Cavanaugh KL, Kripalani S, Rothman RL, Wallston KA. Validation of a Short, 3-Item Version of the Subjective Numeracy Scale. Med Decis Making. 2015;35(8):932-936.

23. Chew LD, Bradley KA, Boyko EJ. Brief questions to identify patients with inadequate health literacy. Fam Med. 2004;36(8):588-594

24. Parker RM, Baker DW, Williams MV, Nurss JR. The test of functional health literacy in adults: a new instrument for measuring patients' literacy skills. $J$ Gen Intern Med. 1995;10(10):537-541.

25. Baker DW, Williams MV, Parker RM, Gazmararian JA, Nurss J. Development of a brief test to measure functional health literacy. Patient Educ Couns. 1999;38(1):33-42. 
26. Pfeiffer E. A short portable mental status questionnaire for the assessment of organic brain deficit in elderly patients. J Am Geriatr Soc. 1975;23(10):433-441.

27. Tong A, Sainsbury P, Craig J. Consolidated criteria for reporting qualitative research (COREQ): a 32-item checklist for interviews and focus groups. Int J Qual Health Care. 2007;19(6):349-357.

28. Formiga F, Chivite D, Sole A, Manito N, Ramon JM, Pujol R. Functional outcomes of elderly patients after the first hospital admission for decompensated heart failure (HF). A prospective study. Arch Gerontol Geriatr. 2006;43(2):175-185

29. van Walraven C, Austin PC, Jennings A, Quan H, Forster AJ. A modification of the Elixhauser comorbidity measures into a point system for hospital death using administrative data. Med Care. 2009;47(6):626-633.

30. Elixhauser A, Steiner C, Harris DR, Coffey RM. Comorbidity measures for use with administrative data. Med Care. 1998;36(1):8-27.

31. Kroenke K, Strine TW, Spitzer RL, Williams JB, Berry JT, Mokdad AH. The $\mathrm{PHQ}-8$ as a measure of current depression in the general population. Journal Affect Disord. 2009;114(1-3):163-173.

32. Zou G. A modified poisson regression approach to prospective studies with binary data. Am J Epidemiol. 2004;159(7):702-706.

33. Bohannon AD, Fillenbaum GG, Pieper CF, Hanlon JT, Blazer DG. Relationship of race/ethnicity and blood pressure to change in cognitive function. J Am Geriatr Soc. 2002;50(3):424-429.

34. Little $R$, Hyonggin A. Robust likelihood-based analysis of multivariate data with missing values. Statistica Sinica. 2004;14:949-968.

35. Harrell FE. Regression Modeling Strategies. New York: Springer-Verlag; 2016.

36. R: A Language and Environment for Statistical Computing. [computer program]. Vienna, Austria: R Foundation for Statistical Computing; 2015.

37. McNaughton CD, Collins SP, Kripalani S, et al. Low numeracy is associated with increased odds of 30-day emergency department or hospital recidivism for patients with acute heart failure. Circ Heart Fail. 2013;6(1):40-46.

38. Abdel-Kader K, Dew MA, Bhatnagar M, et al. Numeracy Skills in CKD: Correlates and Outcomes. Clin J Am Soc Nephrol. 2010;5(9):1566-1573.

39. Yee LM, Simon MA. The role of health literacy and numeracy in contraceptive decision-making for urban Chicago women. J Community Health. 2014;39(2):394-399

40. Cajita MI, Cajita TR, Han HR. Health Literacy and Heart Failure: A Systematic Review. J Cardiovasc Nurs. 2016;31(2):121-130.
41. Pressler SJ, Subramanian U, Kareken D, et al. Cognitive deficits and health-related quality of life in chronic heart failure. J Cardiovasc Nurs. 2010;25(3):189-198.

42. Riley PL, Arslanian-Engoren C. Cognitive dysfunction and self-care decision making in chronic heart failure: a review of the literature. Eur J Cardiovasc Nurs. 2013;12(6):505-511.

43. Woo MA, Macey PM, Fonarow GC, Hamilton MA, Harper RM. Regional brain gray matter loss in heart failure. J Appl Physiol. 2003;95(2):677-684.

44. Levin SN, Hajduk AM, McManus DD, et al. Cognitive status in patients hospitalized with acute decompensated heart failure. Am Heart J. 2014;168(6):917-923.

45. Huynh QL, Negishi K, Blizzard L, et al. Mild cognitive impairment predicts death and readmission within 30 days of discharge for heart failure. Int J Cardiol. 2016;221:212-217

46. Davis KK, Allen JK. Identifying cognitive impairment in heart failure: a review of screening measures. Heart Lung. 2013;42(2):92-97.

47. Tung YC, Chou SH, Liu KL, et al. Worse Prognosis in Heart Failure Patients with 30-Day Readmission. Acta Cardiol Sin. 2016;32(6):698-707.

48. Loop MS, Van Dyke MK, Chen L, et al. Comparison of Length of Stay, 30-Day Mortality, and 30-Day Readmission Rates in Medicare Patients With Heart Failure and With Reduced Versus Preserved Ejection Fraction. Am J Cardiol. 2016;118(1):79-85.

49. Malki Q, Sharma ND, Afzal A, et al. Clinical presentation, hospital length of stay, and readmission rate in patients with heart failure with preserved and decreased left ventricular systolic function. Clin Cardiol. 2002:25(4):149-152.

50. Vader JM, LaRue SJ, Stevens SR, et al. Timing and Causes of Readmission After Acute Heart Failure Hospitalization-Insights From the Heart Failure Network Trials. J Card Fail. 2016;22(11):875-883.

51. O'Connor CM, Miller AB, Blair JE, et al. Causes of death and rehospitalization in patients hospitalized with worsening heart failure and reduced left ventricular ejection fraction: results from Efficacy of Vasopressin Antagonism in Heart Failure Outcome Study with Tolvaptan (EVEREST) program. Am Heart J. 2010;159(5):841-849.e1.

52. Matsuoka S, Kato N, Kayane T, et al. Development and Validation of a Heart Failure-Specific Health Literacy Scale. J Cardiovasc Nurs. 2016;31(2):131-139.

53. Molloy GJ, Johnston DW, Witham MD. Family caregiving and congestive heart failure. Review and analysis. Eur J Heart Fail. 2005;7(4):592-603.

54. Nicholas Dionne-Odom J, Hooker SA, Bekelman D, et al. Family caregiving for persons with heart failure at the intersection of heart failure and palliative care: a state-of-the-science review. Heart Fail Rev. 2017;22(5):543-557. 